UFIFT-HEP-98-19

\title{
The case for a Standard Model with Anomalous $U(1)$
}

\author{
Pierre Ramond \\ Institute for Fundamental Theory, \\ Department of Physics, University of Florida \\ Gainesville FL 32611, USA \\ Invited Talk at Orbis Scientiae, Miami, FL, December $197 n$
}

\begin{abstract}
A gauged phase symmetry with its anomalies cancelled by a Green-Schwarz mechanism, broken at a large scale by an induced Fayet-Iliopoulos term, is a generic feature of a large class of superstring theories. It induces many desirable phenomenological features: Yukawa coupling hierarchy, the emergence of a small Cabibbo-like expansion parameter, relating the Weinberg angle to $b-\tau$ unification, and the linking of R-parity conservation to neutrino masses. Some are discussed in the context of a three-family model which reproduces all quark and lepton mass hierarchies as well as the solar and atmospheric neutrino oscillations.
\end{abstract}




\section{Introduction}

The commonly accepted lore that string theories do not imply robust relations among measurable parameters is challenged in a large class of effective low energy theories derived from string models contain an anomalous $U(1)$ with anomalies cancelled by the Green-Schwarz mechanism [1] at cut-off. As emphasized by 't Hooft long ago, anomalies provide a link between infrared and ultraviolet physics. In these theories, this yields relations between the low-energy parameters of the standard model and those of the underlying theory through anomaly coefficients. An equally important feature is that as the dilaton gets a vacuum value, it generates a Fayet-Iliopoulos that triggers the breaking [2] of the anomalous gauged symmetry at a large computable scale.

Through the anomalous $U(1)$, the Weinberg angle at cut-off is related to anomaly coefficients [3]. A simple model [4] with one family-dependent anomalous $U(1)$ beyond the standard model was the first to exploit these features to produce Yukawa hierarchies and fix the Weinberg angle. It was soon realized that some features could be abstracted from the presence of the anomalous $U(1)$ : expressing the ratio of down-like quarks to charged lepton masses in terms of the Weinberg angle [5, 6, 7], the suppression of the bottom to the top quark masses [8], relating [9] the uniqueness of the vacuum to Yukawa hierarchies and the presence of MSSM invariants in the superpotential, and finally relating the see-saw mechanism [10] to R-parity conservation.

These theories are expressed as effective low-energy supersymmetric theories with a cut-off scale $M$. The anomalous symmetry implies:

- A Cabibbo-like expansion parameter for the mass matrices.

- Quark and charged lepton Yukawa hierarchies, and mixing, including the bottom to top Yukawa suppression.

- The value of the Weinberg angle at unification.

- Three massive neutrinos with mixings that give the small-angle MSW effect for the solar neutrino deficit, and the large angle mixing necessary for the atmospheric neutrino effect.

- Natural R-parity conservation linked to massive neutrinos.

- A hidden sector that contains strong gauge interactions with chiral matter.

An important theoretical requirement is that the vacuum, in which the anomalous symmetry is broken by stringy effects, be free of flat directions associated with the MSSM invariants, and preserve supersymmetry.

The anomalous $U(1)$ also provides a possible explanation of supersymmetry breaking. Since the hidden sector contains a gauge theory with strong coupling, the Green-Schwarz mechanism requires that it have a mixed anomaly as well. 
This implies that the hidden matter is chiral with respect to the anomalous symmetry. As shown by Binétruy and Dudas [11], any strong coupling gauge theory with $X$-chiral fermions breaks supersymmetry (even QCD). A recent analysis 12 improves their mechanism by showing that the dilaton $F$-term does not vanish, providing for gaugino masses and possibly solving the FCNC problem.

In the following, we present the generic features of this type of model, and illustrate some in the context of a realistic three-family model.

\section{Applications to the standard model}

We consider models which have a gauge structure broken in two sectors: a visible sector, and a hidden sector, linked by the anomalous symmetry and possibly other Abelian symmetries (as well as gravity).

$$
G_{\text {visible }} \times U(1)_{X} \times U(1)_{Y^{(1)}} \cdots \times U(1)_{Y^{(M)}} \times G_{\text {hidden }},
$$

where $G_{\text {hidden }}$ is the hidden gauge group, and

$$
G_{\text {visible }}=S U(3) \times S U(2) \times U(1)_{Y} .
$$

is the standard model. Of the $M+1$ extra symmetries,one which we call $X$, is anomalous in the sense of Green-Schwarz.

The symmetries, $X, Y^{(a)}$ are spontaneously broken at a high scale by the Fayet-Iliopoulos term generated by the dilaton vacuum. This DSW vacuum [2] is required by phenomenology to preserve both supersymmetry and the standard model symmetries.

We assume the smallest matter content needed to reproduce the observed quark and charged lepton hierarchy, cancel the anomalies associated with the extra gauge symmetries, and has a unique vacuum structure:

- Three chiral families

- One standard-model vector-like pair of Higgs weak doublets.

- Three right-handed neutrinos $\bar{N}_{i}$,

- Standard model vector-like pairs,

- Chiral fields that are needed to break the three extra $U(1)$ symmetries in the DSW vacuum. We denote these fields by $\theta_{a}$.

- Hidden sector gauge interactions and their matter, together with singlet fields, needed to cancel the remaining anomalies. 


\section{Anomalies}

When viewed from the infrared, the anomaly constraints put strong restrictions on the low energy theory. In a four-dimensional theory, the Green-Schwarz anomaly compensation mechanism occurs through a dimension-five term that couples an axion to all the gauge fields. As a result, any anomaly linear in the $X$-symmetry must satisfy the Green-Schwarz relations

$$
\left(X G_{i} G_{j}\right)=\delta_{i j} C_{i},
$$

where $G_{i}$ is any gauge current. The anomalous symmetry must have a mixed gravitational anomaly, so that

$$
(X T T)=C_{\text {grav }} \neq 0,
$$

where $T$ is the energy-momentum tensor. In addition, the anomalies compensated by the Green-Schwarz mechanism satisfy the universality conditions

$$
\frac{C_{i}}{k_{i}} \equiv \frac{C_{\text {grav }}}{12} \text { for all } i .
$$

A similar relation holds for $C_{X} \equiv(X X X)$, the self-anomaly coefficient of the $X$ symmetry. These result in important numerical constraints, which restrict the matter content of the model. All other anomalies must vanish:

$$
\left(G_{i} G_{j} G_{k}\right)=\left(X X G_{i}\right)=0 .
$$

In terms of the standard model, the vanishing anomalies are therefore of the following types:

- The first involve only standard-model gauge groups $G_{\mathrm{SM}}$, with coefficients $\left(G_{\mathrm{SM}} G_{\mathrm{SM}} G_{\mathrm{SM}}\right)$, which cancel for each chiral family and for vector-like matter. Also the hypercharge mixed gravitational anomaly $(Y T T)$ vanishes.

- The second type is where the new symmetries appear linearly, of the type $\left(Y^{(i)} G_{\mathrm{SM}} G_{\mathrm{SM}}\right)$. If we assume that the $Y^{(i)}$ are traceless over the three chiral families, these vanish over the three families of fermions with standard-model charges. Hence they must vanish on the Higgs fields: with $G_{\mathrm{SM}}=S U(2)$, it implies the Higgs pair is vector-like with respect to the $Y^{(i)}$. It also follows that the mixed gravitational anomalies $\left(Y^{(i)} T T\right)$ are zero over the fields with standard model quantum numbers.

- The third type involve the new symmetries quadratically, of the form $\left(G_{\mathrm{SM}} Y^{(i)} Y^{(j)}\right)$. These vanish by group theory except for those of the form $\left(Y Y^{(i)} Y^{(j)}\right)$. In general two types of fermions contribute: the three chiral families and standard-model vector-like pairs. 
- The remaining vanishing anomalies involve the anomalous charge $X$.

- With $X$ family-independent, and $Y^{(i)}$ family-traceless, the vanishing of the $\left(X Y Y^{(i)}\right)$ anomaly coefficients over the three families is assured: so they must also vanish over the Higgs pair. This means that $X$ is vector-like on the Higgs pair. This is an important result, as it implies that the standard-model invariant $H_{u} H_{d}$ (the $\mu$ term) has zero $X$ and $Y^{(i)}$ charges; it can appear by itself in the superpotential, but we are dealing with a string theory, where mass terms do not appear in the superpotential: it can appear only in the Kähler potential. This results, after supersymmetry-breaking in an induced $\mu$-term, of weak strength, as suggested by Giudice and Masiero 13.

- The coefficients $\left(X Y^{(i)} Y^{(j)}\right), i \neq j$. Since standard-model singlets can contribute to these anomalies, we expect cancellation to come about through a combination of hidden sector and singlet fields.

- The coefficient $(X X Y)$. This imposes an important constraint on the $X$ charges on the chiral families.

- The coefficients $\left(X X Y^{(i)}\right)$; with family-traceless symmetries, they vanish over the three families of fermions with standard-model charges, but contributions are expected from other sectors of the theory.

The building of models in which these anomaly coefficients vanish is highly non-trivial. Finding a set of charges which satisfy all anomaly constraints, and reproduce phenomenology is highly constrained. In the three-family model it will even prove predictive in the neutrino sector.

\subsection{Standard Model Anomalies}

In the standard model, we consider three anomalies associated with its three gauge groups,

$$
C_{\text {color }}=(X S U(3) S U(3)) ; \quad C_{\text {weak }}=(X S U(2) S U(2)) ; \quad C_{\mathrm{Y}}=(X Y Y),
$$

when () stands for the trace. They can be expressed [8] in terms of the $X$-charges of the invariants of the MSSM

$$
\begin{gathered}
C_{\text {color }}=\frac{1}{2} \sum_{i}\left[X_{i i}^{[u]}+X_{i i}^{[d]}\right]-3 X^{[\mu]}, \\
C_{\mathrm{Y}}+C_{\text {weak }}-\frac{8}{3} C_{\text {color }}=2 \sum_{i}\left[X_{i i}^{[e]}-X_{i i}^{[d]}\right]+2 X^{[\mu]},
\end{gathered}
$$

where $X_{i j}^{[u]}$ is the $X$-charge of $\mathbf{Q}_{i} \overline{\mathbf{u}}_{j} H_{u}, X_{i j}^{[d]}$ that of $\mathbf{Q}_{i} \overline{\mathbf{d}}_{j} H_{d}, X_{i j}^{[e]}$ that of $L_{i} \bar{e}_{j} H_{d}$, and finally $X^{[\mu]}$ that of the $\mu$-term $H_{u} H_{d}$, where $i, j$ are the family 
indices. Also the mixed gravitational anomaly over the three chiral families is given by

$$
C_{\text {grav }}^{[\mathrm{fam}]}=3 C_{\text {color }}+\sum_{i} X_{i i}^{[e]}-X^{[\mu]} .
$$

In theories derived from superstrings, the integer level numbers $k_{\text {color }}$ and $k_{\text {weak }}$ are equal, resulting in the equality

$$
C_{\text {weak }}=C_{\text {color }} \text {. }
$$

These imply that, as long as these anomaly coefficients do not vanish, the MSSM Yukawa invariants cannot all appear at tree level, as their $X$-charges are necessarily non-zero. This means that not all Yukawa couplings can be of the same order of magnitude, resulting in some sort of Yukawa hierarchy.

More specific conclusions can be reached by assuming that the $X$ charges are family-independent and the $Y^{(i)}$ are family-traceless. As we have seen, the $\mu$-term has vector-like charges, $X^{[\mu]}=0$.

By further assuming that the top quark Yukawa mass coupling occurs at tree-level, we have $X_{33}^{[u]}=X^{[u]}=0$. This implies that the $X$-charge of the down quark Yukawa is proportional to the color anomaly, and thus cannot vanish: the down Yukawa is necessarily smaller than the top Yukawa, leading to the suppression of $m_{b}$ over $m_{t}$, after electroweak breaking! The non-vanishing of the color anomaly implies the (observed) suppression of the bottom mass relative to the top mass.

The second anomaly equation simplifies to

$$
C_{\mathrm{Y}}-\frac{5}{3} C_{\text {weak }}=6\left[X^{[e]}-X^{[d]}\right],
$$

stating that the relative suppression of the down to the charged lepton sector is proportional to the difference of two anomaly coefficients. The data, extrapolated to near unification scales indicates that there is no relative suppression between the two sectors, suggesting that difference should vanish. Remarkably, the vanishing [3] of that combination fixes the value of the Weinberg angle through the string of relations

$$
\frac{3}{5}=\frac{C_{\mathrm{weak}}}{C_{\mathrm{Y}}}=\frac{k_{\mathrm{weak}}}{k_{\mathrm{Y}}}=\frac{g_{Y}^{2}}{g_{\mathrm{weak}}^{2}}=\tan ^{2} \theta_{w} .
$$

This happens exactly at the phenomenologically preferred value of the Weinberg angle: the $b-\tau$ unification is related to the value of the Weinberg angle [5]!

The application of the Green-Schwarz structure to the standard model is consistent with many of its phenomenological patterns. However, more can be said through a careful study of the DSW vacuum. 


\section{The DSW vacuum}

When the dilaton acquires its vacuum value, an anomalous Fayet-iliopoulos $D$ term is generated through the gravitational anomaly. In the weak coupling limit of the string, it is given by

$$
\xi^{2}=\frac{g^{2}}{192 \pi^{2}} M_{\text {Planck }}^{2} C_{\text {grav }},
$$

where $g$ is the string coupling constant. This induces the breaking of $X$ and $Y^{(i)}$ below the cut-off.

Phenomenology require that neither supersymmetry nor any of the standard model symmetries be broken at that scale. This puts severe restrictions on the form of the superpotential and the matter fields [9].

The analysis of the vacuum structure of supersymmetric theories is greatly facilitated by the fact that the solutions of the vacuum equations for the D-terms are in one-to-one correspondance with holomorphic invariants. This analysis has been recently generalized to include an anomalous Fayet-Iliopoulos term.

In order to get a unique determination of the DSW vacuum, we need as many singlet superfields, $\theta_{a}$, as there are broken symmetries. Only they assume vev's as a result of the FI term. They are standard model singlets, but not under $X$ and $Y^{(a)}$. If more fields than broken symmetries assume non-zero values in the DSW vacuum, we would have undetermined flat directions and hierarchies.

We assemble the charges in a $(M+1) \times(M+1)$ matrix $\mathbf{A}$, whose rows are the $X, Y^{(i)}$ charges of the $\theta$ fields, respectively. Assuming the existence of a supersymmetric vacuum where only the $\theta$ fields have vacuum values, implies from the vanishing of the $M+1 D$-terms

$$
\mathbf{A}\left(\begin{array}{c}
\left|\theta_{1}\right|^{2} \\
\cdot \\
\left|\theta_{M+1}\right|^{2}
\end{array}\right)=\left(\begin{array}{c}
\xi^{2} \\
\cdot \\
0
\end{array}\right) .
$$

For this vacuum solution to exist, the matrix $\mathbf{A}$ must have an inverse and the entries in the first row of its inverse must be positive. The solution to these equations naturally provide computably small expansion parameters $\lambda_{a}=<$ $\left|\theta_{a}\right|>_{0} / M$. In the case when all expansion parameters are the same we can relate their value in terms of standard model quantities

$$
\lambda_{a}=\frac{\alpha}{4 \pi} C_{\text {color }},
$$

where $\alpha$ is the unified gauge coupling at unification.

Another important consequence is that there is no holomorphic invariant polynomial involving the $\theta$ fields alone. Another is that the $\theta$ sector is necessarily anomalous. Indeed, let us assume that it has no mixed gravitational anomalies. This means that all the charges are traceless over the $\theta$ fields. now the $(M+$ 1) $\theta$ fields form a representation of $S U(M+1)$, and the tracelessness of the 
charges insures that they be members of $S U(M+1)$. So we are looking for $M$ non-anomalous symmetries in $S U(M+1)$, which is impossible except for $M=1$. If two or more of the charges are the same on the $\theta$ 's, we could have anomaly cancellation, but then the matrix $A$ would be singular, contrary to the assumption of the DSW vacuum. Hence this sector will in general be anomalous.

For a thorough analysis of the vacuum with FY term, we refer the reader to Ref. [14, 9]. Here, we simply note two striking generic facts of phenomenological import. Consider any invariant $I$ of the MSSM. It corresponds 15] to a possible flat direction of the non-anomalous supersymmetric vacuum. For that configuration, all its fields are aligned to the same vacuum value, as required by the vanishing of the non-anomalous $D$-terms of the standard model symmetries. It follows that the contribution of these terms of the anomalous $D_{X}$ will be proportional to its $X$-charge [16]. In order to forbid this flat direction to appear alone in the vacuum, it is therefore necessary to require that its charge be of the wrong sign to forbid a solution of $D_{X}=\xi^{2}$. This implies a holomorphic invariant of the form $I \mathcal{P}\left(\theta_{a}\right)$, where $\mathcal{P}$ is a holomorphic polynomial in the $\theta$ 's. The $D$-term equations are not sufficient to forbid this flat direction together with $\theta$ fields. We have to rely on the $F$-terms associated with that invariant polynomial, and its presence is needed in the superpotential. Fortunately, phenomenology also requires such terms to appear in the superpotential. This is the first of several curious links between phenomenology and the vacuum structure near unification scales! One can see that the existence of this invariant is predicated on the invertibility of $\mathbf{A}$, the same condition for the DSW vacuum.

The second point addresses singlet fields that do not get vev's in the DSW vacuum. To implement the seesaw mechanism, there must be right-handed neutrinos, $\bar{N}_{i}$. Since they have no vev, their X-charge must also be of the wrong sign, which allows for holomorphic invariants of the form $\bar{N}^{A} \mathcal{P}(\theta)$, where $A$ is a positive integer. The case $A=1$ is forbidden as it breaks supersymmetry. Thus $A \geq 2$. The case $A=2$ generates Majorana masses for these fields in the DSW vacuum. W scale. To single out $A=2$ we need to choose the $X$ charges of the $\bar{N}_{i}$ to be a negative half-odd integers. To implement the seesaw, the right-handed neutrinos couple to the standard model invariants $L_{i} H_{u}$, which requires that $X_{L_{i} H_{u}}$ is also a half-odd integer, while all other MSSM invariants have positive or zero integers $X$-charges.

\section{A Three-Family Model}

We can see how some of the features we have just discussed lead to phenomenological consequences in the context of a three-family model [17, 18], with three Abelian symmetries broken in the DSW vacuum. The matter content of the theory is inspired by $E_{6}$, which contains two Abelian symmetries outside of the 
standard model: the first $U(1)$, which we call $V^{\prime}$, appears in the embedding

$$
E_{6} \subset S O(10) \times U(1)_{V^{\prime}}
$$

The second $U(1)$, called $V$, appears in

$$
S O(10) \subset S U(5) \times U(1)_{V}
$$

The two non-anomalous symmetries are

$$
\begin{aligned}
& Y^{(1)}=\frac{1}{5}(2 Y+V)\left(\begin{array}{ccc}
2 & 0 & 0 \\
0 & -1 & 0 \\
0 & 0 & -1
\end{array}\right) \\
& Y^{(2)}=\frac{1}{4}\left(V+3 V^{\prime}\right)\left(\begin{array}{ccc}
1 & 0 & 0 \\
0 & 0 & 0 \\
0 & 0 & -1
\end{array}\right),
\end{aligned}
$$

The family matrices run over the three chiral families, so that $Y^{(1,2)}$ are familytraceless. Since $\operatorname{Tr}\left(Y Y^{(i)}\right)=0$, there is no appreciable kinetic mixing between the non-anomalous $U(1) \mathrm{s}$.

The $X$ charges on the three chiral families in the $\mathbf{2 7}$ are of the form

$$
X=\left(\alpha+\beta V+\gamma V^{\prime}\right)\left(\begin{array}{lll}
1 & 0 & 0 \\
0 & 1 & 0 \\
0 & 0 & 1
\end{array}\right)
$$

where $\alpha, \beta, \gamma$ are expressed in terms of the $X$-charges of $\bar{N}_{i}(=-3 / 2)$, that of $\mathbf{Q} \overline{\mathbf{d}} H_{d}(=-3)$, and that of the vector-like pair , mass term $\bar{E} E(=-3)$.

The matter content of this model is the smallest that reproduces the observed quark and lepton hierarchy while cancelling the anomalies associated with the extra gauge symmetries:

- Three chiral families each with the quantum numbers of a $\mathbf{2 7}$ of $E_{6}$. This means three chiral families of the standard model, $\mathbf{Q}_{i}, \overline{\mathbf{u}}_{i}, \overline{\mathbf{d}}_{i}, L_{i}$, and $\bar{e}_{i}$, together with three right-handed neutrinos $\bar{N}_{i}$, three vector-like pairs denoted by $E_{i}+\overline{\mathbf{D}}_{i}$ and $\bar{E}_{i}+\mathbf{D}_{i}$, with the quantum numbers of the $\mathbf{5}+$ $\overline{\mathbf{5}}$ of $S U(5)$, and finally three real singlets $S_{i}$.

- One standard-model vector-like pair of Higgs weak doublets.

- Chiral fields that are needed to break the three extra $U(1)$ symmetries in the DSW vacuum. We denote these fields by $\theta_{a}$. In our minimal model with three symmetries that break through the FI term, we just take $a=1,2,3$. The $\theta$ sector is necessarily anomalous.

- Other standard model singlet fields. 
- Hidden sector gauge interactions and their matter.

Finally, the charges of the $\theta$ fields is given in terms of the matrix

$$
\mathbf{A}=\left(\begin{array}{ccc}
1 & 0 & 0 \\
0 & -1 & 1 \\
1 & -1 & 0
\end{array}\right)
$$

Its inverse

$$
\mathbf{A}^{-1}=\left(\begin{array}{ccc}
1 & 0 & 0 \\
1 & 0 & -1 \\
1 & 1 & -1
\end{array}\right),
$$

shows all three fields acquire the same vacuum value.

In the following, we will address only the features of the model which are of more direct phenomenological interest. For more details, the interested reader is referred to the original references [17, 18].

\subsection{Quark and Charged Lepton Masses}

The Yukawa interactions in the charge $2 / 3$ quark sector are generated by operators of the form

$$
\mathbf{Q}_{i} \overline{\mathbf{u}}_{j} H_{u}\left(\frac{\theta_{1}}{M}\right)^{n_{i j}^{(1)}}\left(\frac{\theta_{2}}{M}\right)^{n_{i j}^{(2)}}\left(\frac{\theta_{3}}{M}\right)^{n_{i j}^{(3)}},
$$

in which the exponents must be positive integers or zero. Assuming that only the

top quark Yukawa coupling appears at tree-level, a straighforward computation of their charges yields in the DSW vacuum the charge $2 / 3$ Yukawa matrix

$$
Y^{[u]} \sim\left(\begin{array}{ccc}
\lambda^{8} & \lambda^{5} & \lambda^{3} \\
\lambda^{7} & \lambda^{4} & \lambda^{2} \\
\lambda^{5} & \lambda^{2} & 1
\end{array}\right)
$$

where $\lambda=\left|\theta_{a}\right| / M$ is the common expansion parameter.

A similar computation is now applied to the charge $-1 / 3$ Yukawa standard model invariants $\mathbf{Q}_{i} \overline{\mathbf{d}}_{j} H_{d}$. The difference is that $X^{[d]}$, its $X$-charge does not vanish. As long as $X^{[d]} \leq-3$, we deduce the charge $-1 / 3$ Yukawa matrix

$$
Y^{[d]} \sim \lambda^{-3 X^{[d]}-6}\left(\begin{array}{ccc}
\lambda^{4} & \lambda^{3} & \lambda^{3} \\
\lambda^{3} & \lambda^{2} & \lambda^{2} \\
\lambda & 1 & 1
\end{array}\right) .
$$

Diagonalization of the two Yukawa matrices yields the CKM matrix

$$
\mathcal{U}_{C K M} \sim\left(\begin{array}{ccc}
1 & \lambda & \lambda^{3} \\
\lambda & 1 & \lambda^{2} \\
\lambda^{3} & \lambda^{2} & 1
\end{array}\right)
$$


This shows the expansion parameter to be of the same order of magnitude as the Cabibbo angle $\lambda_{c}$.

The eigenvalues of these matrices reproduce the geometric interfamily hierarchy for quarks of both charges

$$
\begin{array}{lll}
\frac{m_{u}}{m_{t}} \sim \lambda_{c}^{8}, & \frac{m_{c}}{m_{t}} \sim \lambda_{c}^{4} . \\
\frac{m_{d}}{m_{b}} \sim \lambda_{c}^{4}, & \frac{m_{s}}{m_{b}} \sim \lambda_{c}^{2},
\end{array}
$$

while the quark intrafamily hierarchy is given by

$$
\frac{m_{b}}{m_{t}}=\cot \beta \lambda_{c}^{-3 X^{[d]}-6} \text {. }
$$

implying the relative suppression of the bottom to top quark masses, without large $\tan \beta$. These quark-sector results are the same as in a previously published model [17, but our present model is different in the lepton sector.

The analysis in the charged lepton sector proceeds in similar ways. No dimension-three term appears and the standard model invariant $L_{i} \bar{e}_{j} H_{d}$ have $X$-charge $X^{[e]}$. For $X^{[e]}=-3$, there are supersymmetric zeros in the (21) and (31) position, yielding

$$
Y^{[e]} \sim \lambda_{c}^{3}\left(\begin{array}{ccc}
\lambda_{c}^{4} & \lambda_{c}^{5} & \lambda_{c}^{3} \\
0 & \lambda_{c}^{2} & 1 \\
0 & \lambda_{c}^{2} & 1
\end{array}\right)
$$

Its diagonalization yields the lepton interfamily hierarchy

$$
\frac{m_{e}}{m_{\tau}} \sim \lambda_{c}^{4}, \quad \frac{m_{\mu}}{m_{\tau}} \sim \lambda_{c}^{2} .
$$

Our choice of $X$ insures that $X^{[d]}=X^{[e]}$, which guarantees through the anomaly conditions the correct value of the Weinberg angle at cut-off, since

$$
\sin ^{2} \theta_{w}=\frac{3}{8} \quad \leftrightarrow \quad X^{[d]}=X^{[e]}
$$

it sets $X^{[d]}=-3$, so that

$$
\frac{m_{b}}{m_{\tau}} \sim 1 ; \quad \frac{m_{b}}{m_{t}} \sim \cot \beta \lambda_{c}^{3} .
$$

It is a remarkable feature of this type of model that both inter- and intrafamily hierarchies are linked not only with one another but with the value of the Weinberg angle as well. In addition, the model predicts a natural suppression of $m_{b} / m_{\tau}$, which suggests that $\tan \beta$ is of order one. 


\subsection{Neutrino Masses}

Neutrino masses are naturally generated by the seesaw mechanism 10 if the three right-handed neutrinos $\bar{N}_{i}$ acquire a Majorana mass in the DSW vacuum. The flat direction analysis indicates that their $X$-charges must be negative half-odd integers, with $X_{\bar{N}}=-3 / 2$ preferred by the vacuum analysis. Their standard-model invariant masses are generated by terms of the form

$$
M \bar{N}_{i} \bar{N}_{j}\left(\frac{\theta_{1}}{M}\right)^{p_{i j}^{(1)}}\left(\frac{\theta_{2}}{M}\right)^{p_{i j}^{(2)}}\left(\frac{\theta_{3}}{M}\right)^{p_{i j}^{(3)}},
$$

where $M$ is the cut-off of the theory. In the $(i j)$ matrix element. The Majorana mass matrix is computed to be

$$
M \lambda_{c}^{7}\left(\begin{array}{ccc}
\lambda_{c}^{6} & \lambda_{c}^{5} & \lambda_{c} \\
\lambda_{c}^{5} & \lambda_{c}^{4} & 1 \\
\lambda_{c} & 1 & 0
\end{array}\right) .
$$

Its diagonalization yields three massive right-handed neutrinos with masses

$$
m_{\bar{N}_{e}} \sim M \lambda_{c}^{13} ; \quad m_{\bar{N}_{\mu}} \sim m_{\bar{N}_{\tau}} \sim M \lambda_{c}^{7} .
$$

By definition, right-handed neutrinos are those that couple to the standardmodel invariant $L_{i} H_{u}$, and serve as Dirac partners to the chiral neutrinos. In our model,

$$
X\left(L_{i} H_{u} \bar{N}_{j}\right) \equiv X^{[\nu]}=0 .
$$

The superpotential contains the terms

$$
L_{i} H_{u} \bar{N}_{j}\left(\frac{\theta_{1}}{M}\right)^{q_{i j}^{(1)}}\left(\frac{\theta_{2}}{M}\right)^{q_{i j}^{(2)}}\left(\frac{\theta_{3}}{M}\right)^{q_{i j}^{(3)}}
$$

resulting, after electroweak symmetry breaking, in the orders of magnitude (we note $\left.v_{u}=\left\langle H_{u}^{0}\right\rangle\right)$

$$
v_{u}\left(\begin{array}{ccc}
\lambda_{c}^{8} & \lambda_{c}^{7} & \lambda_{c}^{3} \\
\lambda_{c}^{5} & \lambda_{c}^{4} & 1 \\
\lambda_{c}^{5} & \lambda_{c}^{4} & 1
\end{array}\right)
$$

for the neutrino Dirac mass matrix. The actual neutrino mass matrix is generated by the seesaw mechanism. A careful calculation yields the orders of magnitude

$$
\frac{v_{u}^{2}}{M \lambda_{c}^{3}}\left(\begin{array}{ccc}
\lambda_{c}^{6} & \lambda_{c}^{3} & \lambda_{c}^{3} \\
\lambda_{c}^{3} & 1 & 1 \\
\lambda_{c}^{3} & 1 & 1
\end{array}\right)
$$


A characteristic of the seesaw mechanism is that the charges of the $\bar{N}_{i}$ do not enter in the determination of these orders of magnitude as long as there are no massless right-handed neutrinos. Hence the structure of the neutrino mass matrix depends only on the charges of the invariants $L_{i} H_{u}$, already fixed by phenomenology and anomaly cancellation. In particular, the family structure is that carried by the lepton doublets $L_{i}$. In our model, since $L_{2}$ and $L_{3}$ have the same charges, it ias not surprising that we have no flavor distinction between the neutrinos of the second and third family. In models with two non-anomalous flavor symmetries based on $E_{6}$ the matrix $(5.25)$ is a very stable prediction of our model. Its diagonalization yields the neutrino mixing matrix 19]

$$
\mathcal{U}_{\mathrm{MNS}}=\left(\begin{array}{ccc}
1 & \lambda_{c}^{3} & \lambda_{c}^{3} \\
\lambda_{c}^{3} & 1 & 1 \\
\lambda_{c}^{3} & 1 & 1
\end{array}\right),
$$

so that the mixing of the electron neutrino is small, of the order of $\lambda_{c}^{3}$, while the mixing between the $\mu$ and $\tau$ neutrinos is of order one. Remarkably enough, this mixing pattern is precisely the one suggested by the non-adiabatic MSW [20] explanation of the solar neutrino deficit and by the oscillation interpretation of the reported anomaly in atmospheric neutrino fluxes (which has been recently confirmed by the Super-Kamiokande [21] and Soudan [22] collaborations).

Whether the present model actually fits both the experimental data on solar and atmospheric neutrinos or not depends on the eigenvalues of the mass matrix (5.25). A naive order of magnitude diagonalization gives a $\mu$ and $\tau$ neutrinos of comparable masses, and a much lighter electron neutrino:

$$
m_{\nu_{e}} \sim m_{0} \lambda_{c}^{6} ; \quad m_{\nu_{\mu}}, m_{\nu_{\tau}} \sim m_{0} ; \quad m_{0}=\frac{v_{u}^{2}}{M \lambda_{c}^{3}},
$$

The overall neutrino mass scale $m_{0}$ depends on the cut-off $M$. Thus the neutrino sector allows us, in principle, to measure it.

At first sight, this spectrum is not compatible with a simultaneous explanation of the solar and atmospheric neutrino problems, which requires a hierarchy between $m_{\nu_{\mu}}$ and $m_{\nu_{\tau}}$. However, the estimates (5.27) are too crude: since the $(2,2),(2,3)$ and $(3,3)$ entries of the mass matrix all have the same order of magnitude, the prefactors that multiply the powers of $\lambda_{c}$ in (5.25) can spoil the naive determination of the mass eigenvalues. A more careful analysis shows that even with factors of order one, it is possible to fit the atmospheric neutrino anomaly as well. A welcome by-product of the analysis is that the mixing angle is actually driven to its maximum value. We refer the reader to Ref.[18] for more details. The main point of this analysis is that maximal mixing between the second and third family in the neutrino sector occurs naturally as it is determined from the structure of the quark and charged lepton hierarchies. 


\section{R-Parity}

The invariants of the minimal standard model and their associated flat directions have been analyzed in detail in the literature [23]. In models with an anomalous $U(1)$, these invariants carry in general $X$-charges, which, as we have seen, determines their suppression in the effective Lagrangian. Just as there is a basis of invariants, proven long ago by Hilbert, the charges of these invariants are not all independent; they can in fact be expressed in terms of the charges of the lowest order invariants built out of the fields of the minimal standard model, and some anomaly coefficients.

The $X$-charges of the three types of cubic standard model invariants that violate $R$-parity as well as baryon and/or lepton numbers can be expressed in terms of the $X$-charges of the MSSM invariants and the R-parity violating invariant

$$
X^{[\not R]} \equiv X\left(L H_{u}\right)
$$

through the relations

$$
\begin{gathered}
X_{L \mathbf{Q} \overline{\mathbf{d}}}=X^{[d]}-X^{[\mu]}+X^{[\not / R]}, \\
X_{L L \bar{e}}=X^{[e]}-X^{[\mu]}+X^{[\not / h]} . \\
X_{\overline{\mathbf{u}} \overline{\mathbf{d}} \overline{\mathbf{d}}}=X^{[d]}+X^{[/ / /]}+\frac{1}{3}\left(C_{\text {color }}-C_{\text {weak }}\right)-\frac{2}{3} X^{[\mu]} .
\end{gathered}
$$

Although they vanish in our model, we still display $X^{[u]}$ and $X^{[\mu]}=0$, since these sum rules are more general.

In the analysis of the flat directions, we have seen how the seesaw mechanism forces the $X$-charge of $\bar{N}$ to be half-odd integer. Also, the Froggatt-Nielsen [24] suppression of the minimal standard model invariants, and the holomorphy of the superpotential require $X^{[u, d, e]}$ to be zero or negative integers, and the equality of the Kác-Moody levels of $S U(2)$ and $S U(3)$ forces $C_{\text {color }}=C_{\text {weak }}$, through the Green-Schwarz mechanism. Thus we conclude that the $X$-charges of these operators are half-odd integers, and thus they cannot appear in the superpotential unless multiplied by at least one $\bar{N}$. This reasoning can be applied to the higher-order $\not R$ operators since their charges are given by

$$
\begin{aligned}
X_{\mathbf{Q Q Q} H_{d}} & =X^{[u]}+X^{[d]}-\frac{1}{3} X^{[\mu]}-X^{[\not / R]}, \\
X_{\overline{\mathbf{d}} \overline{\mathbf{d}} \overline{\mathbf{d}} L L} & =2 X^{[d]}-X^{[u]}-\frac{5}{3} X^{[\mu]}+3 X^{[\not / R]}, \\
X_{\mathbf{Q Q Q Q} \overline{\mathbf{u}}} & =2 X^{[u]}+X^{[d]}-\frac{4}{3} X^{[\mu]}-X^{[\not / R]}, \\
X_{\overline{\mathbf{u}} \overline{\mathbf{u}} \overline{\bar{u}} \bar{e} \bar{e}} & =2 X^{[u]}-X^{[d]}+2 X^{[e]}-\frac{2}{3} X^{[\mu]}-X^{[\not / R]},
\end{aligned}
$$


It follows that there are no $R$-parity violating operators, whatever their dimensions : through the right-handed neutrinos, $R$-parity is linked to halfodd integer charges, so that charge invariance results in $R$-parity invariance. Thus none of the operators that violate $R$-parity can appear in holomorphic invariants: even after breaking of the anomalous $X$ symmetry, the remaining interactions all respect $R$-parity, leading to an absolutely stable superpartner. This is a general result deduced from the uniqueness of the DSW vacuum, the Green-Schwarz anomaly cancellations, and the seesaw mechanisms.

\section{Conclusion}

The case for an anomalous $U(1)$ extension to the standard model is particularly strong. We have presented many of its phenomenological consequences. In a very unique model, we detailed how the neutrino matrices are predicted. However much remains to be done: the nature of the hidden sector, and supersymmetry breaking. Our model only predicts orders of magnitude of Yukawa couplings. To calculate the prefactors, a specific theory is required. Many of the features we have discussed are found in the context of free fermion theories [25], which arise in the context of perturbative string theory. It is hoped that since they involve anomalies, these features can also be derived under more general assumptions. A particularly difficult problem is that of the cut-off scale. From the point of view of the low energy, there is only one scale of interest, that at which the couplings unify, and the Green-Schwarz mechanism, by fixing the weak and color anomalies, identifies the cut-off as the unification scale. On the other hand, another mass scale appears in the theory through the size of the anomalous FI term, and the two values do not coincide, the usual problem of string unification. It is hoped that the calculation of the Fayet-Iliopoulos term in other regimes will throw some light on this problem.

Our simple model has too many desirable phenomenological features to be set aside, and we hope that a better understanding of fundamental theories will shed light on this problem.

\section{Acknowledgements}

I would like to thank Professor B. Kursunoglu for his kind hospitality and for giving me the opportunity to speak at this pleasant conference, as well as my collaborators, N. Irges and S. Lavignac, on whose work much of the above is based. This work was supported in part by the United States Department of Energy under grant DE-FG02-97ER41029. 


\section{References}

[1] M. Green and J. Schwarz, Phys. Lett. B149 (1984) 117.

[2] M. Dine, N. Seiberg and E. Witten, Nucl. Phys. B289 (1987) 589; J. Atick, L. Dixon and A. Sen, Nucl. Phys. B292 (1987) 109.

[3] L. Ibáñez, Phys. Lett. B303 (1993) 55.

[4] L. Ibáñez and G. G. Ross, Phys. Lett. B332 (1994) 100.

[5] P. Binétruy and P. Ramond, Phys. Lett. B350 (1995) 49; P. Binétruy, S. Lavignac, and P. Ramond, Nucl. Phys. B477 (1996) 353.

[6] Y. Nir, Phys. Lett. B354 (1995) 107.

[7] V. Jain and R. Shrock, Phys. Lett. B352 (1995) 83.

[8] P. Ramond, Kikkawa Proceedings, 1996.hep-ph/9604251

[9] P. Binétruy, S. Lavignac, Nikolaos Irges, and P. Ramond, Phys. Lett. B403 (1997) 38.

[10] M. Gell-Mann, P. Ramond, and R. Slansky in Sanibel Talk, CALT-68709, Feb 1979, and in Supergravity (North Holland, Amsterdam 1979). T. Yanagida, in Proceedings of the Workshop on Unified Theory and Baryon Number of the Universe, KEK, Japan, 1979.

[11] P. Binetruy, E. Dudas, Phys. Lett. B389 (1996), 503. G. Dvali and A. Pomarol, Phys. Rev. Lett. 77, 3728 (1996).

[12] Nima Arkani-Hamed, M. Dine, and S. Martin, hep-ph/9803432.

[13] G. Giudice and A. Masiero, Phys. Lett. B206 (1988) 480; V.S. Kaplunovsky and J. Louis, Phys. Lett. B306 (1993) 269.

[14] N. Irges and S. Lavignac, preprint UFIFT-HEP-97-34, hep-ph/9712239, to be published in Phys. Letters.

[15] F. Buccella, J.-P. Derendinger, S. Ferrara and C.A. Savoy, Phys. Lett. B115 (1982) 375.

[16] E. Dudas, C. Grojean, S. Pokorski and C.A. Savoy, Nucl. Phys. B481 (1996) 85.

[17] John K. Elwood, Nikolaos Irges, and P. Ramond, Phys. Lett. B413 (1997) 322.

[18] N. Irges, S. Lavignac and P. Ramond, UFIFT-HEP-98-06, (1998); hep$\mathrm{ph} / 9802334$. 
[19] Z. Maki, M. Nakagawa and S. Sakata, Prog. Theo. Phys. 28 (1962) 247.

[20] S.P. Mikheyev and A.Yu. Smirnov, Yad. Fiz. 42, 1441 (1985) [Sov. J. Nucl. Phys. 42, 913 (1985)]; Il Nuovo Cimento C 9, 17 (1986); L. Wolfenstein,Phys. Rev. D 17, 2369 (1978); Phys. Rev. D 20, 2634 (1979).

[21] E. Kearns, talk at the ITP conference on Solar Neutrinos: News about SNUs, December 2-6, 1997.

[22] S.M. Kasahara et al., Phys. Rev. D55 (1997) 5282.

[23] T. Gherghetta, C. Kolda and S.P. Martin, Nucl. Phys. B468 (1996) 37.

[24] C. Froggatt and H. B. Nielsen Nucl. Phys. B147 (1979) 277.

[25] A. Faraggi, Nucl. Phys. B387 (1992) 239, ibid. B403 (1993) 101, ibid. B407 (1993) 57. 